\title{
The O-Factor: Aligning Organizational Arrangements with Big Data Analytics Diffusion
}

\author{
Lotte A. L. Elsman \\ University of Amsterdam Business School \\ lotteelsman@hotmail.com
}

\author{
Hans P. Borgman \\ University of Amsterdam Business School \\ h.p.borgman@uva.nl
}

\begin{abstract}
This study aims to better understand how and why organizational arrangements of Big Data Analytics (BDA) evolve over time in established firms. As BDA initiatives grow in scope and importance, organizational arrangements tend to change, with changes impacting the success of the initiative. This study focuses on the importance of four constructs influencing organizational arrangements during BDA diffusion: the analytics structure, the leadership role, the culture, and the employee skills. Propositions derived from the literature guide the analysis of seven case studies of organizations adopting BDA. The findings help to understand BDA diffusion through (1) aligning structure with business value creation, (2) (new) leadership that trusts and shows exemplary usage of BDA, (3) a culture of trust with constant experimentation for business opportunities and (4) more diversified employee roles. A discussion of academic and managerial implications and suggestions for future research completes this study.
\end{abstract}

\section{Introduction}

"You can't manage what you don't measure" [1]. The rise of big data analytics (BDA) has been a central topic of discussion for researchers over the last decade [2], [3], [4], [5], [6] and can be described as "the extensive use of data, statistical and quantitative analysis, explanatory predictive models and fact-based management to drive decisions and actions and to manage the business more effectively" [7]. Although BDA was a relatively new topic a few years ago and most traditional organizations were at early stages of adopting these technologies, it has now become increasingly important in every day's decision processes [2]. The availability of big data changes the way decisions are made in traditional organizations [3] and underlines the need for making decisions based on the analysis of data, rather than on intuitiveness or gut feeling [8]. The more data-driven the organization is, the better the organization performs. Specifically, firms that base their decisions on data are more profitable and there is more productivity in the organization compared to their competitors [8]. While these data-driven transformations are beneficial for organizational results, they imply significant transformations at multiple levels which poses large organizational challenges. As a consequence, it takes a long time for organizations to gain value from BDA initiatives [2], [9]. The current literature focuses mainly on the technical features of BDA with limited focus on the organizational transformations it entails and how this should be leveraged [10]. However, this is crucial as it is found that when organizational arrangements do not support the business's needs, it negatively affects organizations in leveraging BDA [11], [12].

According to a literature review of Vial (2019), changes in the organizational arrangement regarding digital technology adoption include changes in the organizational structure, culture, leadership, and employee roles (Figure 1) [13]. Where Vial (2019) gives a clear overview of the most important literature on digital transformations, he does not make a distinction between different digital technologies while observing changes in their organizational arrangement.

\section{Figure 1: DT Framework}

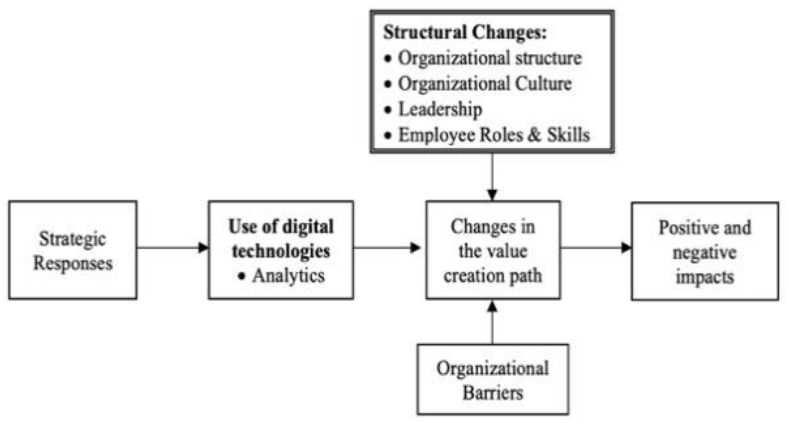


However, it is found that most companies underestimate the difference of BDA from traditional information technology (IT) (i.e., ERP, cloud computing, social media) projects [14].

This research posits that BDA adoption and diffusion is different and dissimilar to traditional IT developments. Even though BDA depends on IT components that offer the tools for accessing and analyzing data resources, the execution and use of BDA is exceptional as it combines data requests with business context to identify patterns for decision support [15]. While IT initiatives are mainly an implementation and adoption challenge, BDA initiatives are an adaption and diffusion (continued usage) challenge [9]. Especially with BDA adoption, no organizational setup is permanent as the BDA diffusion evolves [16].

There is a lack of academic literature investigating how firms are changing their organizational arrangements exclusively to BDA for performance improvement. An understanding of this is especially important for established firms, that need to additionally unlearn many of the existing practices to adapt to newer BDA methods [17].

To address this gap, this study provides an outlook on how and why established firms have diffused BDA across the organization and how with-it changes in the organizational arrangement (structure, leadership, culture, employee roles) have taken place over the years. Our study builds on the BDA diffusion framework of Mikalef et al. (2020) as well as the digital transformation framework of Vial (2019). As Mikalef et al. (2020) acknowledged how inertial forces diffuse during BDA diffusion, this study explores adding the four aspects of an organizational arrangement to the theory. In addition, this research builds on the framework of Vial (2019) by exploring BDA as an exclusive technology rather than seeing the digital technologies as universal. Therefore, the formulated research question for this study is: "How can we understand the transformation of organizational arrangements during big data analytics diffusion within established firms?"

A multiple-case design is guided with seven firms out of different industries. The cases are studied by indepth interviews. Furthermore, collection of the data is navigated by the propositions derived from the literature review, which are discussed in section 2. In section 3, the research method is explained. Hereafter, in section 4 , an outline of the cases is given. Furthermore, in section 5, a cross-case analysis is conducted and presented after which, in section 6, the findings and implications are discussed together with suggestions for future research. By understanding the evolution of BDA, it tells us more about why some organizations fail to realize performance gains and how this can be overcome by implementing changes in organizational arrangements. This also contributes to the managerial relevance of this study as it gives managers an understanding of the organizational changes they need to consider before embarking BDA.

\section{Literature review}

In order to explore how organizational arrangements evolve during the diffusion of BDA, we first discuss the different diffusion phases [2]. Thereafter, the main aspects concerning organizational arrangements are reviewed and propositions are formulated to guide the research and attain the purpose of this study.

\subsection{Big Data Analytics Diffusion}

As shown in Figure 1, besides implementing changes in the organizational setup, firms must simultaneously overcome barriers, including resistance and inertial forces that hinder their transformation effort [13]. Building on this, Mikalef et al. (2020) identified organizational inertial forces as well as how they can be overcome within different phases of BDA diffusion. However, one of the downsides of this research is that organizations expect that BDA investments will pay off once they have overcome these inertial forces and barriers, before BDA has been completely incorporated within the organization with the appropriate organizational arrangements [2].

To understand the diffusion of BDA throughout the organization, the diffusion framework of Mikalef et al. (2020), is based on the technology adoption framework of Mergel and Bretschneider (2013) [2], [18]. Within this framework, three stages of incorporation of BDA in the organization are identified and will be used in this research as a guiding instrument. This framework divides three phases into (1) experimentation, (2) to order-from-chaos to (3) institutionalization. In the first phase, experimenters work to deploy the technology throughout the organization and communicate its value to other departments or units. The trigger can come either from the IT department or from the top management which considers BDA as worth looking into. In the second stage, different units within the organization become acquainted to analytics. The success of using BDA largely depends on the establishment of governance practices for the deployment and use of the technology. Furthermore, in the institutionalization phase, BDA becomes part of the organizational fabric. Commonly, in this phase, there is a well-defined strategy on how BDA is employed on a firm level along with a clear assessment of the expected business value [2]. BDA should be embedded in the core process of an organization since it is strongly related to 
the decision-making process and the business strategy [9]. This means that after the adoption of analytics organizations are required to be able to constantly reorientate and change the setup of organizational components [19].

\subsection{Organizational Arrangements}

This section explores the literature behind the four aspects of organizational arrangements in BDA diffusion from which leading propositions emerge.

\subsubsection{Structure}

The organizational structure has been extensively mentioned as one of the most important barriers that influence BDA projects [20]. Therefore, the adoption of BDA requires new organizational structures [16]. Organizations must ask themselves how organizational analytical structures are aligned in a way that datadriven strategies can be fully achieved. Without suitable organizational structures in place, it is difficult to analyze and collect data across the organization and provide insights to where they are most compulsory [5]. Also, within further studies it is found that one of the most difficult organizational challenges for creating value out of BDA is defining how to structure your analytics function to support the businesses needs for a maximum impact [21]. Especially within more established organizations, organizational structures hinder interdisciplinary collaboration across boundaries [3]. Research shows that an important signal that the organizational structure is not working is the complaint from the data analysts that his or her work has little or no impact and that the business keeps doing what is has been doing [22]. Therefore, organizations must both be stable enough by continuing delivering value in their distinctive way, but at the same time be sufficiently agile and adaptive enough to restructure when their business demands for it [23]. Therefore, it is found that it is essential to incorporate flexibility and scalability into analytics programs with an eye regarding the future [21]. In fact, the analytics unit can be organized in six different ways within the organization.

First, in a decentralized analytics structure, analytics are employed in each department of business functions. This means that resources are only allocated to projects within silos, with no analytics activities outside their business unit or function [9]. Second, in a centralized structure, all data analysts are part of the same corporate unit and analysts are employed to projects with strategic priority. According to Fadler \& Legner (2021), this way of structuring increases the opportunity for economy of scale [5]. Third, the consulting structure is a centralized approach whereby the business units "hire" data analysts for analytics projects throughout the organization. Fourth, within the functional model, data analysts are placed within the specific function that dominate analytical activity within an organization. Fifth, within the Centre of Excellence model (COE), analysts are allocated to business units throughout the organization. There is a central entity that coordinates the activities of the business units. Lastly, the federated model describes teams with fundamental data and analytics roles that manage data management and analytics. This type of structuring allows organizations to have both a decentralized and a centralized structure [5].

In the current literature, the pros and cons of the different structures are widely discussed [5], [21], [24]. For example, Davenport (2010) identifies a centralized design as more mature. However, to be able to have an enterprise-wide BDA structure, other researchers conclude that a federated model is needed [5], [24]. Furthermore, the literature highlights that it is key to maintain the organization flexible to encounter the changing needs and expectations of the business [21]. Therefore, it is important that it is formed in such a way that it "keeps analysts both close to the business and close to each other" [25]. Other researchers conclude that none of these structures is perfect and that there is no "correct" way to build an analytics organization that is the right fit for the business [21], [24]. The structure is extremely dependent of organizational characteristics like, size, industry, and data \& analytics ambitions. Organizations that have different priorities and goals may have different organizational structures. This is in line with the contingency theory which suggests that there is no universal best way to organize the analytics effort but that it should fit the organization's environment [26]. Differences in the structures of organizations depends on differences in the technology they employ [27] such that the analytics organization should develop and evolve over time in line with the businesses need [21]. Subsequently, we suppose that implementing and diffusing BDA is different compared to earlier IT innovations as BDA involves constant exploration of BDA insight by experimentation to increase business value.

Proposition 1: The structure of analytics impacts the diffusion of BDA whereby flexibility in structuring BDAteams is crucial to constantly act in line with value creation for the business.

\subsubsection{Leadership}

In addition to the transformation of the organizational analytical structure, extant literature highlights the role of the leadership as another important aspect in ensuring that analytics technologies are properly leveraged and aligned. Since it is found that structuring your analytics effort is context dependent, 
such that contingent structural forms become ineffective or unsustainable, it becomes a managerial task to ensure a structural fit with the organization's goal at each point in time [27]. Executive leaders must keep an ear to the ground for complaints from both the business and the data analysts [22]. For example, if an organization was to employ a centralized analytics structure but the organization has become passive to needs of the business, leaders must rethink the structure that supports the business needs [21]. By designing organizational structures that support a BDA transformation, leaders should be aware of the mechanisms of coordination that are established among different organizational units regarding the data initiatives [28]. Organizations must be structured in a way that data-driven capabilities are effectively and optimally allocated, managed, coordinated in a way that it supports the organizational structure [16], [23]. However, as a leader to be able to restructure the organization and to foster close collaboration between business and IT functions, having trust in the BDA effort is crucial [13]. In the literature it is found that only one-third of CEOs trusts the use of BDA for decision making [29]. In addition, leadership is considered an important factor to establish the degree to which an organization becomes data-driven and an important determinant for the long-term organizational benefits of data-driven decision making [30]. Therefore, to be able to make these right decisions and take the right actions with regard to BDA, leaders must ensure that they have both managerial and technical knowledge and skills [23]. Another important finding from the literature is that leaders must share this knowledge amongst decision makers to get a sustainable competitive differentiation through BDA [31] and showcase the visibly use of BDA to spread the value across the organization [32]. Leaders must continuously communicate and explain the value of BDA, manage the important resources including data and analysts, and create a BDA collaborative culture of knowledge sharing [30], [31].

Proposition 2: Leadership impacts the diffusion of BDA whereby knowledgeable leaders must communicate enthusiasm about BDA and above all act upon value creation through $B D A$.

\subsubsection{Culture}

In addition to the crucial role of the leadership for understanding organizational arrangements in BDA diffusion, the literature highlights the organizational culture as another important construct for effectively diffusing BDA [16]. Especially for established firms, encouraging a culture that supports BDA is difficult compared to many start-ups that have created datadriven cultures from the start.
With the adoption of BDA, a common theme across the literature points to the need for organizations to encourage a willingness to take risks and to experiment with analytics on a small scale before expanding these experiments to the rest of the organization. In doing so, organizations must promote a culture where learning through small, incremental, and iterative change is established, while maintaining their ability to adapt long-term plans [33]. This corresponds to findings in another research describing that organizational agility of adaptiveness and efficiency were identified as the main organizational characteristics for BDA success [34]. Thereby, it also describes that a culture of collaboration must be established to be successful with BDA [31]. However, a critical and continuing issue for established firms is the slow pace in which these firms make the change to an organizational wide data-driven culture [35]. Eventually, to effectively leverage BDA, factbased decision making must be embedded in the organizational culture. This implies that organizations need employees and managers who recognize the importance of data and its analysis [3].

In short, a fact-based decision-making culture is required where the business is running by the numbers and there is experimentation to see what works best in collaboration with the analytics effort [36]. Therefore, changes regarding the organizational analytical structure need to be anchored in the organizational culture. However, this also applies the other way around as the literature shows that the organizational structure impacts the organization towards getting a more datadriven culture [11].

Proposition 3: For BDA diffusion, a transformation into a risk-taking and exploratory culture of fact-based decision making in the BDA team is needed for constant business value creation.

\subsubsection{Employee roles \& skills}

To diffuse BDA, an effective analytics structure requires requestors and users of analytics to collaborate and balance the supply and demand for analytics skills. Namely, changes to the analytics structure and culture lead employees to assume roles that were traditionally outside of their functions [13]. More specifically, analytics enables new forms of decision-making processes which demands the need to develop skills of existing workers as well as hiring new people with the right skills that are essential for the future. BDA technologies are wide in scope and involve multiple skills and expertise. As a result, within BDA diffusion, it is found that there are shifts in the type of tasks that are required to be carried out, as well as the number of tasks. This results in roles that are becoming more diversified and digitally induced. [10]. Accompanying employees through this transition poses significant 
challenges. Thereby, companies must believe and move on in the new reality: technology skills are no longer completely centered in IT: they need to be "marbled" across organizational functions and businesses to achieve transformation success [37]. The relevance of big data and technical skills as well as that of a good understanding of the business unit domain is necessary for BDA success. Furthermore, the literature highlights the importance of a linking person who can bridge the IT and business departments in BDA [3].

Proposition 4: The employee skills impact the diffusion of BDA whereby all roles within the organization must become more data induced and more diversified with both IT and business knowledge.

\section{Research method}

The primary purpose of this study is to acquire a deeper understanding of the transformation of organizational arrangements during BDA diffusion within large, established firms from different industries. As prior research concluded that a broader selection of cases is necessary [11], and to increase reliability and validity of the study [38], [39], a collection of seven cases was selected and reviewed in-depth. All cases have implemented BDA into their decision making for already three years or more. The cases are anonymized at the demand of the interviewed organizations. Prior to the actual data collection, one pilot interview was conducted with a consultant in the field of digital transformation to get a deeper understanding of the topic in practice. The primary data collection method for this research consisted of fourteen semi-structured interviews. The interviews were supplemented by documents provided by the case organization to further validate the evidence [40]. The interview protocol was structured around four main topics, which are the stated propositions as examined in the previous sections and personalized to the role of the interviewee. The unit of analysis were one to three individual employees, working at different levels in the organizations: 1) one senior leader and/or 2) one middle manager of analytics team and in all cases 3) one data analyst/scientist (Table 1). In total, fourteen interviews were conducted online by using Zoom. The interviews lasted between 40-60 minutes and were recorded, transcribed, analyzed, and coded by using NVivo software. To check the objectivity of the coding scheme and improve reliability, the coding of interviews from two cases was analyzed by an independent analyst.

\section{Cases}

As mentioned before, this study focuses on large, established organizations. The seven cases concern organizations that have been actively adopting BDA into their decision-making process for at least three years of more. In table one, firm characteristics are specified for each case study such as the industry, the profession of the interviewees, details of their analytics structure and years of analytics adoption.

Table 1: Case study overview

\begin{tabular}{|c|c|c|c|c|}
\hline Case & Industry & Profession Interviewees & $\begin{array}{l}\text { Years BDA } \\
\text { Adoption }\end{array}$ & $\begin{array}{l}\text { Analytical } \\
\text { Structure }\end{array}$ \\
\hline \multirow[t]{3}{*}{1} & \multirow[t]{3}{*}{ Banking } & Analytics Consultant & \multirow[t]{3}{*}{9} & \multirow[t]{3}{*}{ Federated } \\
\hline & & Global Director & & \\
\hline & & Head of Analytics & & \\
\hline \multirow[t]{2}{*}{2} & \multirow[t]{2}{*}{ Production } & Senior Online Marketeer & \multirow[t]{2}{*}{4} & \multirow[t]{2}{*}{ Decentralized } \\
\hline & & Head of Digital & & \\
\hline \multirow[t]{2}{*}{3} & \multirow{2}{*}{$\begin{array}{l}\text { Consumer } \\
\text { goods }\end{array}$} & Commercial Director & \multirow[t]{2}{*}{5} & \multirow{2}{*}{$\begin{array}{l}\text { Consulting } \\
\text { Model }\end{array}$} \\
\hline & & Business Analyst & & \\
\hline \multirow[t]{2}{*}{4} & \multirow[t]{2}{*}{ Telecom } & Head of Analytics & \multirow[t]{2}{*}{7} & \multirow[t]{2}{*}{ Centralized } \\
\hline & & Analytics Consultant & & \\
\hline \multirow[t]{2}{*}{5} & \multirow[t]{2}{*}{ Oil \& Gas } & IT Manager & \multirow[t]{2}{*}{8} & \multirow[t]{2}{*}{ Federated } \\
\hline & & Business Analyst & & \\
\hline \multirow[t]{2}{*}{6} & \multirow[t]{2}{*}{$\begin{array}{l}\text { Logistics/ } \\
\text { Shipping }\end{array}$} & $\begin{array}{l}\text { Program Manager } \\
\text { Advanced Analytics }\end{array}$ & \multirow[t]{2}{*}{4} & \multirow[t]{2}{*}{ Federated } \\
\hline & & Data Analyst & & \\
\hline 7 & Insurance & $\begin{array}{l}\text { Program Manager } \\
\text { Analytics }\end{array}$ & 4 & $\begin{array}{l}\text { Functional } \\
\text { Model }\end{array}$ \\
\hline
\end{tabular}

\section{Cross-Case Analysis}

In this chapter, the empirical findings will be discussed by a cross-case analysis to compare the results of each proposition (Table 2). Findings are observed and compared to eventually answer the research question.

Table 2: Cross-case analysis

\begin{tabular}{l|c|c|c|c|c|c|}
\hline Case & \multicolumn{3}{|c|}{ P1 } & P2 & P3 & P4 \\
\hline 1 & DC & $\rightarrow$ & FS & $+/+$ & $+/+$ & $+/+$ \\
\hline 2 & DC & - & - & $+/-$ & $+/-$ & $+/-$ \\
\hline 3 & DC & $\rightarrow$ & COS & $+/+$ & $+/+$ & $+/-$ \\
\hline 4 & DC & $\rightarrow$ & CS & $+/+$ & $+/+$ & $+/+$ \\
\hline 5 & DC & $\rightarrow$ & FS & $+/+$ & $+/-$ & $+/+$ \\
\hline 6 & DC & $\rightarrow$ & FS & $+/+$ & $+/+$ & ++ \\
\hline 7 & DC & $\rightarrow$ & FM & $+/-$ & $+/-$ & $+/-$ \\
\hline
\end{tabular}

Note $:$ DC = decentralized structure $; \mathrm{CS}=$ centralized structure $;$ COS = consulting structure; FS = federated structure; FM = Functional Model Note: $\rightarrow=$ transformed into

Note: $+/+=$ supported/implemented; $+/-=$ supported/not yet implemented; -/- not supported, not implemented

Partly supported; - = Not supported

\section{P1: The structure of analytics}

This section highlights that the structure of analytics impacts the diffusion of BDA whereby organizational flexibility is crucial to constantly enable value creation for the business. Overall, we found support for the first 
proposition across the cases as six out of the seven cases have transformed their analytics structure to be able to diffuse BDA. Furthermore, all the cases are constantly investigating how to optimally structure their analytics function to effectively support the business needs. For example, an interviewee from case 1 clearly stated: "It's nice to have a federated structure, but if there is no trust and the team of analysts has to fight for its right to exist at the business-side, at some point it will stop, and it won't be used effectively enough". Furthermore, an interviewee from case 4 also confirms that an analytics structure only works if it supports the needs of the business by stating: "I really believe in the centralization, but we really need the business in this." This is in line with the stated proposition which describes that the analysts' team must be structured in a way closely to the business and close to each other [25].

Furthermore, we have found that there is no universal best way to organize your analytics function. The structure of analytics is highly dependent on the organizational characteristics. However, we do see a similarity between the larger organizations (case 1, 5, 6) which reflect both a centralized and a decentralized structure, also recognized as a federated structure. An interviewee from case 5 explains that as follows: "You want to have a central overview with analysts as they want to compare themselves with other teams of the organization. But it is also the case that certain departments may be interested in things but are not given the opportunity to build them or invest in them centrally. There may still be value in for a department but perhaps not for the rest of the organization. So, there is a balance. But it should not be totally decentralized, because then every department will be doing the same thing and that is a waste of effort". However, in case 7 an interviewee mentioned that all forms of organizational structures regarding data analytics can be found. This is because the different departments are not strongly steered by hierarchical organization but by the functional organization. Therefore, organizational chains can organize their analytics the way they think works best. From this, we can conclude that BDA diffusion can lead organizations toward more than one form of structuring analytics which appears to be more common in larger organizations (1000+ employees).

Furthermore, we found a similarity between all the cases that they all started their analytics adoption within decentralized structure which supports the requisite that organizations need organizational flexibility to transform over time. Case 2, for example, is still in the early stages of BDA diffusion where steps with analytics and data are being taken within the departments but do not transcend the departments. BDA is used more on ad hoc basis instead of having outlined structures in which this is automatically shared throughout the organizations. However, the goal is to eventually move towards centralization as one interviewee stated: "The future will be to develop a single point of truth. To bring all the data together so we have it all in one place". Moreover, within case 5, there also have been a transformation from a more decentralized structure to a more data-sharing structure by stating: "It's very interesting to see where in the beginning everyone sat in decentralization and thought, this data is mine, which transformed to a larger scale and now understands how important it is centralized and shared throughout the organization". In addition, transforming to a more centralized structure is also an important structural change for case 3 and 4 . For example, case 3 which has transformed to one central team of analysts that act as internal consultants for the business unit, whereby one interviewee clearly stated: "We are now really one team, which provides the business with the tools to be able to make data driven decisions. We try to be a kind of internal consultant for the business". However, the advantages of a centralized structure differ per case as an interviewee from case 6 indicates that in a centralized structure it often occurs that the busines is not sufficiently involved with the content.

We found support that the structure of analytics impacts the diffusion of BDA. However, if the structure does not support the business, flexibility is required to adapt while diffusing throughout the organization. In order to make effective changes regarding the structure, the role of leadership, the culture and employee skills are crucial and will therefore be discussed in the following sections.

\section{P2: Leadership}

This section highlights that the leadership impacts the diffusion of BDA whereby knowledgeable leaders must communicate trust in BDA and above all act upon value creation through BDA. Thereby, all the cases confirm that the role of leadership plays an essential role for the diffusion of BDA.

First, we found that organizations with leadership which trusts BDA are further along in the BDA diffusion process (case 1, 3, 4, 5, 6) compared to the organizations that have leadership that does not yet recognize the full potential of BDA (case 2, 7). To support these findings and to better understand BDA diffusion, quotes derived from the interviews will be used as an illustration. For example, an interviewee from case 1 clearly mentioned the importance of trust from the leadership by stating: "I really want to emphasize that our leader plays a very important role in $B D A$, because if he showed resistance then I can use my team with analysts and say well we have a very good team here, but if the leader resists, nothing will come of 
out of that data". Similarly, an interviewee from case 2 indicates the importance of trust within the leadership. However, he stated that their top management has not yet reached the stage to make changes in their organizational arrangement to further diffuse BDA as there is not sufficient trust: "This morning, I presented to the top management to convince them that data and analytics is important, but they still have their doubts about whether this will work and whether it is really possible. It all starts with trust and the vision from the top management that they say: we understand it, go organize it more centrally". Furthermore, the results show that the organizations that have created trust, are able to generate a larger impact since the analytics teams grow in the organizational structure. To support this finding, an interviewee from case 4 clearly stated: "We as a department are growing now that we really have created trust within the top management layer and have proven that we do good things".

Additionally, the interviews from case 1, 3, 4 and 6 show that management must carry out this trust to the organization by communicating the value creation through BDA. For example, an interviewee from case 3 clearly stated: "If you are not a leader who dares to speak out that data plays an important role, then it's not going to be a success within the organization either".

In addition, and relating to the proposition, interviewees from case 1, 2, 3, 4 and 6 mentioned that the top management must have the right knowledge regarding BDA. An interviewee from case 1 stated that particularly leadership must have an understanding in IT by saying: "What's so great with us is that we have a leader who is hugely IT savvy, so he's constantly working with the head of the COE to constantly think about: What is the next step? What else can we do by using the data in the future?" Contrary to case 1, within case 3, one interviewee emphasizes that especially having business knowledge is important for a leader to diffuse BDA by stating: "The leadership must have a very deep understanding of the business, otherwise you can never lead the data-driven transformation". Furthermore, having sufficient knowledge to lead the BDA transformation seems also crucial as it is found that the leadership must act upon value creation from BDA by constantly show exemplary usage of BDA. For example, an interviewee from case 5 stated: "I work with two leadership teams and we also ask them: how are you going to make better decisions based on data in your leadership meetings? That is very important". Thereby, an interviewee from case 2 confirmed this as well: "Also, in the management layers they have to breathe it and make it the core of the business".

Furthermore, we found another important outcome from the interviews regarding the leadership which adds to the stated proposition and earlier literature. In interviews from case 1, 2, 3 and 4 is mentioned that the establishment of new leadership is an important change to be able to facilitate the diffusion of BDA through the organization. One interviewee from case 4 clearly stated that: "Three years ago, we got a new commercial director, and he is very data-driven. I do notice that since he's been here, that we're much more concerned with data. He is a bit of the helicopter view". This is expressed in case 1 as well by stating: "I do see the revision of the strategy and the new CHRO as a kind of acceleration. Now it's all data and digital what is going on". From this, we can argue that in the early phases enthusiasm is important and in the later phases trust helps to diffuse BDA.

\section{P3. Culture}

This section highlights that for BDA diffusion, a transformation into a culture of trust in fact-based decision making is needed including constant exploration for further value creation. From the interviews with case 1, 2, 4, 6 and 7 it is found that the culture around BDA starts with an explorative, learningfrom-failure culture. For example, case 1 started their BDA project by stating the following: "In 2013, my boss said: shall we just do a data and analytics research, just try and see, and then tell the business what we have found, that was a lot of missionary work to the business actually". However, to be able to persist this explorative culture as well as transforming to a culture where evidence-based decision making and constant change is fully trusted throughout the organization, the findings show that all the cases go through a long process. Hence, case 1, 2, 3, 5 and 7 mentioned the culture change as one of the most difficult challenges for diffusing BDA throughout the organization. Thereby, the most challenging is the collaboration with the business and creating trust as the analytics function. For example, an interviewee from case 1 stated: "That's what's all about. That's the first battle that must be fought, that the business trusts the results that come out of the system". This is also expressed in case 4 where an interviewee stated: "Previously, we built a model and then we would go to someone in the business and say: your work can be done in an hour instead of a day. Then they said: I'd rather use my excel because I know what happens in it". She continued by stating: "That is really a culture change, which is often underestimated". From this it becomes clear that organizational arrangements regarding BDA must transform hand-in-hand with the business units, which causes cultural challenges i.e., trust between the business and the data analysts/scientists and a lack of understanding between them. Therefore, a cultural transformation in the business is needed whereby an interviewee from case 3 stated the following: "Data always tells you to a certain 
degree of reliability, something that makes it a factor in decision making. But if you don't have transparent and cultural change management program around that, then it fails in the business as well'. The same counts for case 5 where an interviewee stated: "Doing good analytics is not an IT problem. IT has to give the foundation, the technology, but it takes a very big business transformation to set this up properly". This all corresponds to the proposition, stating that a transformation into a culture of trust in fact-based decision making is mandatory. Namely, changes in the structure can bring the team of analysts and the business closer together, but the culture and the mindset of the employees must support this, otherwise the structure will not be effective. This is also confirmed by the following quote from an interviewee from case 3: "Data-driven is of course a very interesting thing, because that is something that is actually inhuman, but what can make people better in an organization, you start to collaborate better and make decisions together. But if you don't embed that properly in that human and culture, then you're never going to get an analyticsdriven organization".

\section{P4. Employee Roles \& Skills}

This section highlights that the employee skills impact the diffusion of BDA. All roles in the organization must become more data infused and diversified with both IT and business knowledge. Technology skills are no longer centralized in IT and need to be "marbled" across organizational functions and businesses for BDA diffusion [32]. This is supported by cases $1,3,5$ and 6 . To support this finding an interviewee of case 1 by stated: "2, 3 years ago we introduced a curriculum that allowed people within IT to specialize in certain parts of the profession. It's only now that you're seeing the repercussions on the business side. In the beginning a lot was done with data, but it remained in the background for the business side. But slowly they are going to have to work with it as well. This change requires that we also implement the same change on the business term of employee skills". The same applied to case 5, whereby one interviewee stated: "IT is no longer IT, you get more of a blend. It doesn't make sense to say to IT architects, this is what we need and go build it. It's more iterative and specific. In the business, they also need IT capabilities". As cases 1 and 5 are both large organizations, it seems that especially the diversification of roles plays a crucial role for organizations that are already using analytics on a more enterprise level. Moreover, other cases confirm that digital skills are becoming more crucial in BDA diffusion.

For example, within case 1 is, as they are still in the starting phase with BDA, it appears that by having only few people with the right roles and skills, they cannot make sufficient impact on the business side. An interviewee stated: "With the use of digitization and data you just need different skills and that's a very different type of profile than the people who are active within our company". He continued by stating that: "Important to me is that there should be more people with data knowledge within our company. On one hand, that's just good for the continuity, but on the other hand, these are people who can also work with me to make steps. So that we can take people with us and explain them and seek the connection with the people who are less versed in this digitalization. That's easier when there's more of people with digital knowledge".

\section{Discussion and future research}

This study contributes to our understanding of the transformation of organizational arrangements through BDA diffusion and addresses the research question: "How can we understand the transformation of organizational arrangements during big data analytics diffusion within established firms?"

According to our analysis, established firms require a transformation across all four aspects to ensure an organization-wide impact with BDA. The first proposition regarding the structure of the analytics function shows that there is no universal way to structure the analytics function but highly depends on organizational characteristics and requires organizational flexibility. Additionally, and most importantly, structure needs constantly to be aligned with business value creation [5], [24]. Redesigning the analytics function to business processes must be done from a value-creation perspective, rather than just for connecting and automating existing business processes as in traditional IT initiatives.

The second proposition regarding the importance of leadership for BDA diffusion resonates strongly across all cases. Our findings add to the literature regarding responses to overcome BDA challenges [2], [11] as we found that a great acceleration in BDA diffusion is achieved when new leaders are appointed to lead the transformation. We found that new leaders with relevant knowledge and skills are perceived as inspiring and convincing. Another finding that is still little reflected in the literature regarding the role of leadership for BDA diffusion, is that these leaders act upon the value creation by exemplary and visible usage of BDA in addition to sharing knowledge to decision makers and communicating the value about it across the organization [30], [31].

For the third proposition regarding the culture, the findings show that the cultural change is the most challenging obstacle in BDA diffusion. Thereby, most 
importantly, a transformation to a culture of trust at the business side is often overlooked in established firms. Furthermore, and adding to the literature of Mikalef (2020), we found that a culture of exploration should not only be in the initial phase of BDA adoption [2] but must be continuously present during the diffusion of BDA across the organization to explore, in collaboration with the business, new insights and opportunities for the business.

For the fourth and last proposition regarding the employee roles, we found support that employee roles and skills regarding BDA become more important and diversified between the analytics team and the business within larger organizations (case 1, 3, 4, 6). A key transformation is that analytics skills are no longer centered in IT, but that the entire organization must have the understanding and skills to be able to successfully diffuse BDA. This is confirmed by the fact that cases that are still at the beginning of the diffusion (case 2 and 7), lack sufficient numbers of people with the right knowledge and skills needed to really make an impact on the business side.

To conclude, this research demonstrated, and adds to the literature [2], [13], that to be able to diffuse BDA throughout the organization, all constructs impact each other in moving forward to the diffusion phases. It seems that making changes in one aspect, lead toward changes in others as well. Namely, without an effective structure that does not support the culture or a culture that does not support the structure, BDA will not create value for the organization. For this, an effective (new) leader is necessary at the forefront together with sufficient employees with the right knowledge and skills. Our supported findings provide academic contribution as it shows how organizational arrangements regarding structure, culture, leadership, and employee roles apply in BDA diffusion in addition to overcoming organizational barriers and inertial forces [2]. However, future research must further investigate the influences between the constructs.

The implications of this research are threefold. First, the results provide a descriptive explanation of seven in-depth case studies and show the value of making (interlinked) changes in organizational arrangement towards BDA diffusion. As past literature has highlighted the need for research on non-technical aspects of BDA, this research sheds light on the most critical elements and the kind of changes that are required in these elements to ensure an impactful BDA diffusion.

Second, this study also contributes to the body of literature on leadership, structures, culture in relation to BDA in established firms which is different than traditional IT adoption. Most importantly, the findings emphasize the critical role of (new) leadership.
Therefore, we call attention to the development or hiring new leaders with a deep understanding in BDA as well as the right business knowledge who especially show exemplary usage of BDA and share this knowledge with decision makers [31].

Third, this study also contributes to the practice as it guides practitioners in their understanding that BDA diffusion should not be managed in an isolated way as a technical project.

This research does not come without certain limitations. First, the number of interviews, being two to three per organization, could have be expanded if time had permitted. In addition, although we have adopted a multiple case study approach, a broader selection of cases out different industries should be expanded to be able to identify comparisons or differences in changes by organizational context. Last, the BDA diffusion framework of Mikalef (2020) has been used as a guiding tool rather than indicating per phase which concrete changes in the organizational arrangements have taken place. These limitations encountered in this research shed light on potential areas for future research. Furthermore, as it is found that the way analytics teams are structured within organizations is highly dependent on the organizational characteristics, future research based on a broad selection of cases from different industries would need to be investigated to see differences between industries in more detail and to discover what contextual factors influence different organizational arrangements. Furthermore, as Mikalef (2020) suggested to research what conditions firms cause to not opt BDA, it would be interesting to see if the implementation of the described changes are obstacles for not adopting BDA [2].

\section{References}

[1] A. McAfee, E. Brynjolfsson, "Big data: The Management Revolution.," Harv. Bus. Rev., no. October, pp. 1-9, 2012

[2] P. Mikalef, R. van de Wetering, J. Krogstie, "Building dynamic capabilities by leveraging big data analytics: the role of organizational intertia", Information \& Management, 2020

[3] C. Dremel, M. Herterich, J. Wulf, J. Waizmann, \& W. Brenner, "How AUDI AG Established Big Data Analytics in Its Digital Transformation," MIS Q. Executive, 16, 2017

[4] J. Espinosa, F. Armour, "The Big Data Analytics Gold Rush: A Research Framework for Coordination and Governance", Proceedings of the 49th Hawaii International Conference on System Sciences, 11121121,2016

[5] M. Fadler, \& C. Legner, "Toward big data and analytics governance: redefining structural governance mechanisms", Proceeding of the $54^{\text {th }}$ Hawaii 
International Conference on System Sciences, 56965705, 2021

[6] M. Yasmin, E. Tatoglu, H. S. Kilic, S. Zaim, \& D, Delen, "Big data analytics capabilities and firm performance: An integrated MCDM approach", Journal of Business Research, 114, 1-15, 2020

[7] T.H. Davenport, J.G, Harris. "Competing on Analytics: The New Science of Winning". Boston, Mass: Harvard Business School Press, 2007.

[8] F. Provost, T. Fawcett, Data Science for Business, Van Duuren Media, 2013

[9] D. Nam, J. Lee, \& H. Lee, "Business analytics adoption process: An innovation diffusion perspective", International Journal of Information Management, 49, 411-423, 2019

[10] P. Mikalef, I. O. Pappas, J. Krogstie, M. Giannakos, “Big data analytics capabilities: a systematic literature review and research agenda", Information Systems and eBusiness Management, 16, 547-578, 2018

[11] M. Storm, \& H.P. Borgman, "Understanding Challenges and Success Factors in Creating a Data-Driven Culture", Proceedings of the $53^{\text {rd }}$ Hawaii International Conference on System Sciences, 2020

[12] H.W., Khanagha, S., Baden-Fuller, C., Mihalache, O.R., Birkinshaw, "Strategizing in a digital world: Overcoming cognitive barriers, reconfiguring routines and introducing new organizational forms", Long Range Planning, 2021

[13] G. Vial, "Understanding digital transformation: A review and a research agenda." J. Strateg. Inf. Syst. 28, 118-144, 2019

[14] S. Ransbotham, D. Kiron, P.K. Prentice, "Beyond the hype: the hard work behind analytics success", MIT Sloan Management Review, 2016

[15] H. Chen, R.H.L. Chiang, V.C. Storey, "Business Intelligence and Analytics: From Big Data to Big Impact", MIS Quarterly 36(4):1165-1188, 2012

[16] G. Macías-Lizaso Miranda, "Building an effective analytics organization”, McKinsey \& Company, 2018

[17] M.A. Cusumano, "How traditional firms must compete in the sharing economy", Communications of the ACM, 58(1): 32-34, 2015

[18] I. Mergel, S.I. Bretschneider, "A Three-Stage Adoption Process for Social Media Use in Government”, Public Administration Review, 2013

[19] A. Hanelt, R. Bohnsack, D, Marz, C.A. Marante, "A Systematic Review of the Literature on Digital Transformation: Insight and Implications for Strategy and Organizational Change", Journal of Management Studies, 2020

[20] A.A.F Alalawned, S.F. Alkhatib, "The barriers to big data adoption in developing economies", Electron $\mathrm{j}$ inf syst devctries, 2021

[21] J. Griffin, \& T. H. Davenport, "Organizing Analytics From the inside out Establish an analytics ecosystem that drives value throughout the enterprise", Deloitte, 2014

[22] O. Fleming, T. Fountaine, N. Henke, T. Saleh, "Ten red flags signalling your analytics program will fail", McKinsey \& Company, 2018
[23] P. Mikalef, J. Krogstie, I.O. Pappas, \& P. Pavlou, "Exploring the relationship between big data analytics capability and competitive performance: The mediating roles of dynamic and operational capabilities," Information \& Management, 57(2), 103169, 2020

[24] J. Baijens, T. Veldstra, \& R. Helms, "Towards a framework for data analytics governance mechanisms", Twenty-Eighth European Conference on Information Systems - A Virtual AIS Conference, 1-16, 2020

[25] J. Hernandez, B. Berkey, R. Bhattacharya, "Building an Analytics-Driven Organization”, Accenture, 2013

[26] L. Donaldson, "The Contingency Theory of Organizations", SAGE Publications, 2001

[27] M.S. Dijksterhuis, F.A.J. Van den Bosch, H.W. Volberda, "Where Do New Organizational Forms Come From? Management Logics as a Source of Coevolution", Organizational Science, 519-690, 1999

[28] A. Kennedy, T. Gomes, \& O. Bittner, "How to Navigate Data-Driven Transformation", Accenture, 2019

[29] J. Schneider, J.P. Handali, J. vom Brocke, "Increasing trust in (Big) Data Analytics", Advanced Information Systems Engineering Workshops, pp. 70-84, 2018

[30] B. Windt, H. Borgman, C. Amrit, "Understanding leadership challenges and responses in data-driven transformations", Proceedings of the 52nd Hawaii International Conference on System Sciences, 2019

[31] O. Marjanovic, "A novel mechanisms for business analytics value creation: improvement of knowledgeintensive business processes", Journal of Knowledge Management, 2021

[32] T. H. Davenport, N. Mittal "How CEOs Can Lead a Data-Driven Culture", Harvard Business Review, 2020

[33] S. L. Feinzig \& N. Guenole, "Data-driven Cultures Start at the Top", Harvard Business Review, 2020

[34] N. Côrte-Real, T. Oliveira, R. Ruivo, "Assessing business value of Big Data Analytics in European firms", Journal of Business Research 70, 379-390, 2017

[35] T.H. Davenport, R. Bean, "Big Companies are Embracing Analytics, But Most Still Don't Have A Data-Driven Culture", Harvard Business Review, 2018

[36] Watson, J. Hugh, "Tutorial: Big Data Analytics: Concepts, Technologies, and Applications," Communications of the Association for Information Systems, 34, Article 65, 2014

[37] Gartner, "Lack of Skills Threatens Digital Transformations", 2020

[38] K. M. Eisenhardt, "Building Theories from Case Study Research Published by: Academy of Management," vol. 14, no. 4, pp. 532-550, 1989.

[39] R.K. Yin, "Case Study Research: Design and Methods", SAGE Publications, 2009

[40] G.A. Bowen, "Document Analysis as a Qualitative Research Method", Qualitative Research Journal, 9(2), 27-40, 2009

[41] V. Grover, R. H. L. Chiang, T. P. Liang, \& D. Zhang, "Creating Strategic Business Value from Big Data Analytics: A Research Framework", Journal of Management Information Systems, 35(2), 388-423, 2018 